\title{
Ionic Thermoelectric Figure of Merit for Charging of Supercapacitors
}

Hui Wang, Dan Zhao, Zia Ullah Khan, Skomantas Puzinas, Magnus J onsson, Magnus Berggren and Xavier Crispin

The self-archived version of this journal article is available at Linköping University Electronic Press:

http:// urn.kb.se/ resolve?urn=urn:nbn:se:liu:diva-137393

N.B.: When citing this work, cite the original publication.

Wang, H., Zhao, D., Ullah Khan, Z., Puzinas, S., J onsson, M., Berggren, M., Crispin, X., (2017), Ionic Thermoelectric Figure of Merit for Charging of Supercapacitors, ADVANCED ELECTRONIC

MATERIALS, 3(4), 1700013. https:/ / dx.doi.org/10.1002/ aelm.201700013

Original publication available at:

https:// dx.doi.org/ 10.1002/aelm.201700013

Copyright: Wiley: 12 months

http://eu.wiley.com/WileyCDA/ 


\section{Ionic Thermoelectric Figure of Merit for charging of supercapacitors}

Hui Wang", Dan Zhao\#, Zia Ulla Khan, Skomantas Puzinas, Magnus P. Jonsson, Magnus Berggren and Xavier Crispin*

Laboratory of Organic Electronics (LOE), Department of Science and Technology (ITN), Linköping University, SE-60174 Norrköping, Sweden

* Contact E-mail: xavcr@itn.liu.se

\# Those two authors have contributed equally.

Keywords: ionic thermoelectric effect, polyelectrolyte, supercapacitor

Thanks to natural heat gradients, for example, generated by the sun, and the large amount of heat being wasted in combustion engines (35-40\% efficiency), there is a strong interest in heat-to-electricity conversion to contribute in powering our modern society. Thermoelectric applications are typically based on thermodiffusion of electronic charge carriers (electrons or holes) in semiconductors, semimetals or metals. This thermoelectric effect is also referred to as the electronic Seebeck effect. ${ }^{[1]}$ More recently, thermodiffusion of ionic charge carriers in an electrolyte was used to convert heat to electrical energy stored in a supercapacitor. This new device concept, together with the discovery that some electrolytes can provide ionic Seebeck coefficients that are hundred times larger than the electronic Seebeck coefficient of good thermoelectric materials, ${ }^{[2]}$ motivates a fair comparison between electronic and ionic thermoelectric devices.

The electronic Seebeck coefficient $\alpha_{e}$ of a material is defined as the ratio between the open circuit potential $V_{\text {oc }}$ and the temperature difference $\Delta T$ (compensated for the Seebeck coefficient of the metal contacts). If electrons and holes thermo-diffuse towards the colder side at identical rate no thermovoltage is generated. Hence, a non-negligible Seebeck coefficient is obtained for materials with different conductivities for electrons and holes. This is illustrated for a material displaying majority hole $\left(\mathrm{h}^{+}\right)$conduction in Figures 1a and 1b. The electronic Seebeck effect provides the basic principle of operation for thermoelectric generators (TEGs), which can provide a continuous output current and power (Figure 1c). The efficiency of the heat-to-electricity conversion is directly related to $\sqrt{1+Z T_{\mathrm{e}}}$, where $Z T_{\mathrm{e}}$ is the dimensionless thermoelectric figure-of-merit, as introduced by A. F. Ioffe already in 1949. ${ }^{[4]} Z T_{e}\left(=\sigma_{e} \alpha_{e}^{2} T / \lambda\right)$ is defined by three fundamental properties of the thermoelectric material: the electrical conductivity $\sigma_{e}$, the Seebeck coefficient $\alpha_{e}$ and the thermal conductivity $\lambda$. Today, there is an intense strive to optimize the interplay between those three properties and to maximize $Z T_{\mathrm{e}}{ }^{[1]}$ While the major effort is to achieve TEGs based on inorganic materials $\left(Z T_{e}=1.2\right.$ at $300 \mathrm{~K}$ for $\mathrm{Bi}_{2} \mathrm{Te}_{3}$ alloys), ${ }^{[5]}$ recent studies also include oxides, carbon based componds, ${ }^{[6,7]}$ and electronically conducting organic polymers entirely based on atomic elements of high natural abundance $\left(Z T_{e}=0.2-0.4\right.$ at $300 \mathrm{~K}$ for PEDOT) ${ }^{[8,9,10]}$ This opens up for mass production of thermoelectric modules using high-volume printing and extrusion technologies. ${ }^{[8]}$ 
We now move from electronic to ionic electronic thermoelectric materials. Figure $1 \mathrm{~d}$ shows an example of an ionic conductor (that is not electrochemically active, thus excluding any contribution from thermogalvanic effects) ${ }^{[11]}$ that favors the transport of cations over anions when exposed to a thermal gradient. The Soret effect induces ionic concentration differences that generate a thermo-voltage. The ionic Seebeck voltage $\alpha_{i}$ of the ionic conductor is measured as the open circuit voltage $V_{\text {oc }}$ established between the two metal electrodes exposed to different temperatures (assuming a negligible Seebeck coefficient of the metal contacts). ${ }^{[12]}$ The ionic thermoelectric effect occurs in ionic solids, ${ }^{[13,}{ }^{14]}$ liquids or gel electrolytes ${ }^{[15,16]}$ as well as in inorganic solid electrolytes. ${ }^{[17]}$ The thermodiffusion depends on the details of the ion-solvent interactions and it is measured as the so-called heat of transport $\mathrm{Q}^{*}$, which determines the direction and magnitude of thermo-diffusion. ${ }^{[18]}$ The contribution from one type of ions to the ionic Seebeck coefficient can be presented $\alpha=\mathrm{Q}^{*} / N_{\mathrm{a}}|e| T$, where $N_{\mathrm{a}}$ is Avogadro's number and $|e|$ is the charge of an electron. ${ }^{[19]}$ From this, the $\alpha_{\mathrm{i}}$ contribution of diluted ions in water is expected to be in the range of $0.1 \mathrm{mV} / \mathrm{K}$. Analogous to the Seebeck voltage for electronic materials, if cations and anions thermodiffuse at the similar rates, one can foresee that $\alpha_{\mathrm{i}}$ is low. Hence, different mobility or concentration for cations and anions should favor a large $\alpha_{\mathrm{i}}$. Surprisingly, large ionic Seebeck coefficient up to about $10 \mathrm{mV} / \mathrm{K}$ has been measured for asymmetric electrolytes in organic solvent. ${ }^{[20]}$ The origin of this large Seebeck coefficient is not yet fully understood.

One important difference between the ionic Seebeck effect and the electronic Seebeck effect in energy conversion applications is that thermo-diffused ions cannot pass into an external circuit when reaching a metal electrode (Figure 1e). The ionic thermoelectric effect is therefore not suitable for continuous operation of traditional thermoelectric generators. Instead, ions will accumulate at the metal electrodes to form an electric double layer (EDL), and induce a transient thermo-induced current that decreases to zero over time (Figure 1f). The integrated current represents the charge stored in the EDL capacitors located along the metal electrodeelectrolyte interface. ${ }^{[21]}$ When using suitable (high capacitance) electrode materials, the accumulated charge could be dramatically enhanced and this principle can then be used to charge a supercapacitor ${ }^{[2,3]}$ or a battery. ${ }^{[22]}$ In this way, the ionic thermoelectric effect can convert thermal energy to stored electrical energy that later can be used to power an external circuit on demand. When used to charge a supercapacitor, the device is referred to as an ionic thermoelectric supercapacitor (ITESC). ${ }^{[2,3]}$ We note that thermoelectric effects in general are useful for low power energy harvesting, and therefore suitable to slowly charge a battery or a supercapacitor (SC) followed by rapid release and usage of the stored electrical energy as a high power pulse. Hence, the equivalent circuit of the serial connection of a TEG and a SC is one of the key to consider also for thermoelectric applications that are based on electronic materials. Here, we discuss charging efficiency of such circuit, for both electronic and ionic thermoelectric materials. In particular, we demonstrate that the efficiency of the ITESC can be expressed using an ionic thermoelectric figure of merit of the electrolyte $Z T_{\mathrm{i}}=\sigma_{\mathrm{i}} \alpha_{\mathrm{i}}^{2} T / \lambda$, where $\sigma_{\mathrm{i}}$ is the ionic electrical conductivity, $\alpha_{\mathrm{i}}$ is the Seebeck coefficient, and $\lambda$ the thermal conductivity. This relationship will greatly aid the search for suitable ionic thermoelectric materials, by establishing a map of various families of ionic conductors classified by their $Z T_{\mathrm{i}}$ and calculating their efficiency and energy stored per degree of temperature difference.

The ITESC utilizes thermodiffusion of ionic charge carriers to charge a supercapacitor and was recently demonstrated as suitable for intermittent heat sources, ultimately the sun. ${ }^{[2,3]}$ As an example of application, the ITESC could store electrical charge during daytime due to heating by the sun, followed by usage of the energy 
during night by discharging the device. The basic operational protocol of the ITESC is illustrated in Figure $2 .{ }^{[2]}$ (i) First, a heater is switched on to establish a $\Delta T$ across the electrode-electrolyte-electrode stack, resulting in a thermovoltage reaching $V_{\text {thermo }}=\alpha_{\mathrm{i}} \Delta T$ after a certain time of stabilization ( $\left.t_{\mathrm{st}}\right)$. (ii) By connecting the two electrodes, $V_{\text {thermo }}$ charges the supercapacitor. The integrated current during charging represents the charge $Q_{\mathrm{ch}}$ stored at the electrodes of the ITESC. (iii) After charging, the circuit is disconnected (open circuit) and the heater is switched off. In this cooling step, the thermovoltage decays to zero, such that the open circuit potential is entirely governed by the voltage drop (of opposite sign) from the stored charge along the electrode/polyelectrolyte interface of the ITESC. (iv) Finally, the device can be discharged by connecting the ITESC to an external circuit. The integrated discharge current corresponds to the charge $Q_{\text {dis }}$. Recently, Suk Lae Kim et al. successfully made ITESC with similar polyelectrolytes, they observed no leakage current, and the stored charge could be maintained longer than $24 \mathrm{~h}$.For the sake of simplicity in our theoretical description of efficiency and energy, we assume that there is no leakage current and no parasitic self-discharge processes, which leads to $Q_{c h}=\frac{1}{2} C V_{\text {thermo }}^{2}=Q_{d i s}$

Before discussing conversion efficiency and stored energy, it useful to first resolve what are relevant values for the thermoelectric properties of ionic materials. Here, we provide a study on a well-known polymer electrolyte, polystyrene sulfonate sodium ( $\mathrm{PSS}^{-} \mathrm{Na}^{+}$), and we control the ionic mobility by controlling the humidity, in other words by introducing various amount of water. PSS:Na serves as a polyanionic membrane since the negative charge (sulfonate) is attached covalently to the polymer chain and is effectively immobile while the cations are the only mobile species. PSS:Na films with thickness of about $1.5 \mu \mathrm{m}$ were prepared by drop-casting on glass substrates with pre-patterned Au electrodes. The Ionic Seebeck coefficient $\alpha_{i}$ was measured by applying alternating temperature differences $\Delta T$ of increasing magnitude from - $4 \mathrm{~K}$ to $4 \mathrm{~K}$ and following the evolution of the thermovoltage (open circuit voltage) $V_{\text {thermo }}$ (the structure of the setup is shown in supplementary information Figure S1). We find a linear relation between $V_{\text {thermo }}$ and $\Delta T$, and $\alpha_{\mathrm{i}}$ is obtained as the slope of the linear fit to the experimental data (after subtraction of the Seebeck coefficient of the Au electrodes, see Figure S2). At 100\% RH, PSS:Na possesses a large and positive ionic Seebeck coefficient of $+4 \mathrm{mV} / \mathrm{K}$ (Figure 3b). This indicates that PSS:Na is a cation selective conductor, as expected since the polymer anions are essentially immobile because of their large size (Figure 3a). We measured $\alpha_{i}$ at various levels of RH in the range from $50 \%$ to $100 \%$ (Figure 3b). Lowest $\alpha_{i}$ of only $0.26 \mathrm{mV} / \mathrm{K}$ was measured at $50 \% \mathrm{RH}$, followed by an increase with increasing humidity, reaching $4 \mathrm{mV} / \mathrm{K}$ at $100 \% \mathrm{RH}$. The evolution of the ionic conductivity $\sigma_{i}$ versus humidity levels is also given in Figure 3b. The ionic conductivity was characterized by a standard method established for electrochemical devices, ${ }^{[23]}$ as detailed in the supplementary information (Figure S3). The ionic conductivity is lowest at $50 \% \mathrm{RH}$ $(0.026 \mathrm{~S} / \mathrm{m})$ and then increases rapidly to slowly saturate around $80 \% \mathrm{RH}$, finally reaching $1.18 \mathrm{~S} / \mathrm{m}$ at $100 \% \mathrm{RH}$. These results are in agreement with the fact that cations in dry polyelectrolyte films to a great extent are immobile, since they are localized by electrostatic interaction with the polyelectrolyte chains. With increasing humidity, absorbed water molecules in the hygroscopic polyelectrolyte film screen the ions by forming a solvation shell. ${ }^{[17]}$ As a result, the electrostatic attraction between the mobile counter ions and the immobile polyions is suppressed and, thus, the activation energy for transport of counter ions decreases. ${ }^{[24]}$ By further increasing the humidity level, continuous water percolation paths, i.e. conduction channels inside the polyelectrolyte film, are created. Finally, at some elevated RH level, the ionic conductivity saturates and reaches a value similar to those measured in pure aqueous solution. ${ }^{[25]}$ The fact that both $\sigma_{\mathrm{i}}$ and $\alpha_{\mathrm{i}}$ increase with $\mathrm{RH}$ corroborates that ionic diffusion is the main 
origin of the resulting thermo-voltage. ${ }^{[26]}$ Here, we would like to emphasize that $\alpha_{i}$ for completely hydrated PSS:Na films $(100 \% \mathrm{RH})$ is about 40 times larger than typical values for conventional high-performing electronic thermoelectric materials. ${ }^{[12]}$ The power factor, which is defined as $\sigma_{\mathrm{i}} \alpha_{\mathrm{i}}{ }^{2}$ can be calculated to $19 \mu \mathrm{Vm}^{-1} \mathrm{~K}^{-2}$ at $100 \%$ RH.

The thermal conductivity of the PSS:Na film at different RH were measured using the $3 \omega$-method, ${ }^{[27]}$ (see experimental details in SI, Figure S4 and S5). Figure 3c shows that the thermal conductivity $\lambda$ of the polyelectrolyte films increases from $0.35 \pm 0.02 \mathrm{Wm}^{-1} \mathrm{~K}^{-1}$ at $50 \% \mathrm{RH}$ to $0.49 \pm 0.03 \mathrm{Wm}^{-1} \mathrm{~K}^{-1}$ at $100 \% \mathrm{RH}$. This increase is attributed to an increase in the water content of the films. Water swells the hygroscopic polymer film ${ }^{[28]}$ and nanosized aqueous domains and percolation pathways are created. In turn, this increases the net heat transport for the two major transport mechanisms: the phonon transport through the polymer chains and the local convection of water molecules within aqueous-rich domains $\left(\lambda_{\mathrm{H} 2 \mathrm{O}}=0.6 \pm 0.031 \mathrm{Wm}^{-1} \mathrm{~K}^{-1}\right) .{ }^{[29]}$ Finally, the evolution of $\lambda$ and $Z T_{\mathrm{i}}$ versus RH are both presented in Figure 3c. $Z T_{\mathrm{i}}$ increases versus humidity, from $1.55 \times 10^{-6}$ at $50 \% \mathrm{RH}$ to 0.012 at $100 \%$ RH (at room temperature). Hence, varying the humidity has been a useful tool to explore the range of thermoelectric properties available for solid state polymer electrolyte since the $Z T_{\mathrm{i}}$ varies by more than four orders of magnitude.

With this knowledge about the ionic thermoelectric properties of the polyelectrolyte, we now turn to the expected impact when used in thermoelectric energy conversion devices. By using the concept of ITESC, heat is converted to electrical energy stored at the electrodes of the supercapacitor. In a simplified picture, we can evaluate the ITESC using an equivalent circuit based on an internal electric generator providing a voltage $V_{\text {thermo }}$ in series with an ideal supercapacitor of capacitance $C$ and a resistor $R_{\mathrm{s}}$ (Figure $4 \mathrm{a}$ ). This highly resembles a TEG connected in series with a supercapacitor; ${ }^{[2]}$ which will enable a comparison between the energy and charging efficiency for ITESC and TEG-SC using respectively $\left(\alpha_{\mathrm{i}}, Z T_{i}\right)$ and $\left(\alpha_{\mathrm{e}}, Z T_{e}\right)$. The charge stored on the electrodes is $Q_{c h}=$ $C V_{\text {thermo }}=C \alpha_{i} \Delta T$ (Eq. 1) and the stored electrical energy is $E_{c h}=\frac{1}{2} \frac{Q_{c h}{ }^{2}}{C}=\frac{1}{2} C V_{\text {thermo }}{ }^{2}$ (Eq. 2). It is clear from these equations that $E_{\mathrm{ch}}$ should increase linearly and quadratically with the Seebeck coefficient. Assuming a supercapacitor with $C=1 \mathrm{mF}$ and a temperature difference of $\Delta \mathrm{T}=10 \mathrm{~K}$, the stored energy in the ITESCs with the PSS:Na electrolyte increases from $0.004 \mu \mathrm{J}$ to $0.6 \mu \mathrm{J}$ when humidity increased from $50 \%$ to $100 \%$, as shown in Figure 4b. By considering charging of an equivalent supercapacitor connected to a TEG leg (Figure S6. b), we can calculate and compare the stored energy $E_{\mathrm{ch}}$ also for electronic thermoelectric materials. The stored energy is 500 and 3000 times lower respectively for $\mathrm{Bi}_{2} \mathrm{Te}_{3}$ and PEDOT than PSS:Na at RH 100\%. This pinpoints the key advantage of high Seebeck coefficients for ionic thermoelectric materials compared to electronic thermoelectric materials. We also report $E_{\mathrm{ch}}$ for other types of ionic thermoelectric materials in order to establish a first map of ionic thermoelectric materials, including $\mathrm{PEO}-\mathrm{NaOH}^{[2]}, \mathrm{PSSH}^{[3]}$ and ionic liquids. ${ }^{[21]}$

We will now discuss how the efficiency of the ITESC depends on material parameters expressed as a function of $Z T_{\mathrm{i}}$. The efficiency of the total heating-cooling cycle is the electrical energy produced divided by the thermal energy passing through the device. There are two major contributions to the thermal energy. ${ }^{[25]}$ The first is the heat used to warm up the materials, which is proportional to the masses and the heat capacitances of the electrodes and the electrolyte. The second contribution corresponds to the heat absorbed in the device by phenomena similar to traditional thermoelectric generators: ${ }^{[30]}$ (1) Peltier heat absorption from the hot side to the 
cold side due to the thermoelectric current $\left(\alpha_{i} T_{H} \int I_{c h} d t\right.$, where $T_{\mathrm{H}}$ is the temperature at hot side); (2) Joule heating that heats the hot side of the device $\left(\frac{1}{2} \int I_{c h}{ }^{2} R_{S} d t\right)$, where $R_{\mathrm{S}}$ corresponds to the internal ionic resistance of the electrolyte; and (3) the heat that flows along the ITESC due to the temperature difference $\left(\lambda A \int \Delta T d t\right)$, where $A$ is the cross-sectional area of the film in the direction perpendicular to the current. We neglect the first contribution which is highly dependent on the geometry of the device, and focus on the material aspect for the first step. Moreover, the initial heating of the materials is also possible to be reused to some extent in more complex device system. Here maximum heat-to-stored electricity conversion efficiency of an ITESC $\left(\eta_{\Delta \mathrm{Tch}}\right)$ is defined as the ratio between the electrical energy that is generated and stored in the ITESC $\left(E_{\mathrm{ch}}\right)$ during the charging time $\left(t_{\mathrm{ch}}\right)$, and the heat absorbed during charging $\left(Q_{\mathrm{in}}\right), \eta_{\mathrm{ch}}=E_{\mathrm{ch}} / Q_{\mathrm{in}}$. For a specific $t_{\mathrm{ch}}$, the heat input to the hot junction of the ITESC is: ${ }^{[25]}$

$$
Q_{\text {in }}=\alpha_{i} T_{H} \int_{0}^{t_{c h}} I_{c h} d t+\lambda A \int_{0}^{t_{c h}} \Delta T d t-\frac{1}{2} \int_{0}^{t_{c h}} I_{c h}^{2} R_{S} d t
$$

By recognizing that $\int_{0}^{t_{c h}} I_{c h} d t=Q_{c h}=C \alpha_{i} \Delta T$ (Eq. 4) and $\int_{0}^{t_{c h}} I_{c h}{ }^{2} R_{s} d t=C\left(\alpha_{i} \Delta T\right)^{2}$ (Eq. 5), Eq. 3 is reduced to:

$Q_{\text {in }}=\alpha_{i} T_{H} C \alpha_{i} \Delta T+\lambda A \Delta T t_{c h}-\frac{1}{4} C\left(\alpha_{i} \Delta T\right)^{2} \quad$ (Eq. 6)

Using the time constant for charging the supercapacitor as $\tau=C R_{\mathrm{S}}\left(R_{S}=\frac{L}{A \sigma_{i}}\right)$ and $5 \tau$ as the charging time (99\% of the charge has been transferred during this period), the heat absorbed can be expressed as:

$Q_{\text {in }}=\alpha_{i} T_{H} C \alpha_{i} \Delta T+\frac{5 C \lambda \Delta T}{\sigma_{i}}-\frac{1}{4} C\left(\alpha_{i} \Delta T\right)^{2} \quad$ (Eq. 7)

The maximum charging efficiency of ITESC $\left(\eta_{\Delta \mathrm{Tch}}\right)$ can now be expressed as:

$\eta_{\Delta \mathrm{Tch}}=\frac{\Delta T}{2 T_{H}+\frac{10 \lambda}{\sigma_{i} \alpha_{i}^{2}}-\frac{1}{2} \Delta T} \quad$ (Eq. 8)

It is clear from Eq. 8 that the maximum charging efficiency is closely related to the same parameters that determines the ionic thermoelectric properties: $\sigma_{i}, \alpha_{i}$ and $\lambda$. Similar to classic electronic materials, the efficiency will increase with higher ionic conductivity and Seebeck coefficient and lower thermoconductivity. The dependence of these three parameters on the maximum efficiency of ITESC is illustrated in Figure S7 by fixing two of the parameters and vary the third (at $\Delta T=10 \mathrm{~K}$ and $T_{\mathrm{H}}=303 \mathrm{~K}$ ). This will give useful insights into the importance of the individual properties, while we point out that they are typically not uncorrelated for real materials. As reasoned in the introduction, it would be highly valuable if $\eta_{c h}$ could also be directly evaluated based on $Z T_{i}$. Indeed $Z T_{\mathrm{i}}=\sigma_{\mathrm{i}} \alpha_{\mathrm{i}}^{2} T / \lambda$ as defined above, allows us to modify Eq. 8 to:

$\eta_{c h}=\frac{\Delta T}{T_{H}} \frac{Z T_{i}}{2 Z T_{i}+\frac{10 T}{T_{H}}-\frac{1}{2} Z T_{i} \frac{\Delta T}{T_{H}}}$

As comparison, the maximum efficiency for traditional electronic thermoelectric generator (TEG) is:

$$
\Phi_{\max }=\frac{\Delta T}{T_{H}} \frac{\sqrt{1+Z T_{e}}-1}{\sqrt{1+Z T_{e}}+\frac{T_{C}}{T_{H}}}
$$


For material with the same ZT value, the maximum efficiency from the classic TEGs is higher than from ITESC. This is due to the fact that the output power is not constant in ITESC, instead decrease with time.

Figure 4c shows the dependence of ZT for the efficiency of ITESCs composed of different materials, as well as for the equivalence of electronic TEGs connected to a SC (at $T=298 \mathrm{~K}, \Delta T=10 \mathrm{~K}, C=1 \mathrm{mF}$ ). Because $Z T$ varies dramatically with humidity for PSS: $\mathrm{Na}$, the efficiency also spans a large range, from $5 \times 10^{-7} \%$ to $0.004 \%$. It is worth mentioning that the efficiency of PSS:Na at high humidity is larger than other ionic thermoelectric materials that have higher ionic Seebeck coefficients, which can be explained by the higher ionic conductivity in the aqueous PSS:Na gels. ${ }^{[31]}$ Fig. 4c shows that the ionic material with the highest efficiency is PSSH ${ }^{[3]}$ due to its high ionic conductivity. Indeed the proton mobility in aqueous system is known to be much higher than sodium cation through efficient transport mechanism such as the Grotthus mechanism at high humidity ${ }^{[24]}$. Hence, ionic thermoelectric material can reach efficiencies similar to electronic thermoelectric material for charging supercapacitor with alternative heat sources. The exciting point is that there has been a lot of research on reaching high ionic conductivity, but it remains to reveal how to optimize the ionic Seebeck coefficient in polymer electrolytes. Hence, for that parameter, there is vast potential for improvement.

Finally, we stress the important finding that the charging efficiency of ITESC can be expressed based on an ionic figure of merit $Z T_{\mathrm{i}}$, in analogy to traditional TEGs. Because $Z T_{\mathrm{i}}$ includes the relevant thermoelectric properties of the material, this enables a direct prediction of efficiency with new improved materials developed in future research. Moreover, for the first time, the energy conversion efficiency of ionic and electronic thermoelectric material is presented together with the same meaning through the equivalence of a ITESCs with a series circuit TEG-SC. This builds the foundation for a new route to develop the technology in case of energy harvesting from intermittent heat sources.

\section{Acknowledgements}

The authors acknowledge the European Research Council (ERC-starting-grant 307596), the Knut and Alice Wallenberg foundation (project “Tail of the sun”), The Swedish Energy Agency, the Advanced Functional Materials Center at Linköping University, the Wenner-Gren Foundations, the Swedish Research Council, the Swedish Foundation for Strategic Research, the ÅForsk Foundation, and the Swedish Government Strategic Research Area in Materials Science on Functional Materials at Linköping University (Faculty Grant SFO-MatLiU No 2009 00971).

\section{References}

$[1]$

[2]

[3]

[4]

[5]

[6]
G. J. Snyder, E. S. Toberer, Nat Mater 2008, 7, 105.

D. Zhao, H. Wang, Z. U. Khan, J. Chen, R. Gabrielsson, M. P. Jonsson, M. Berggren, X. Crispin, Energ. Environ. Sci. 2016, 9, 1450.

S. L. Kim, H. T. Lin, C. Yu, Adv. Eng. Mater. 2016, 6, 160546.

M. Vedernikov, E. lordanishvili, "AF loffe and origin of modern semiconductor thermoelectric energy conversion", presented at Thermoelectrics, 1998. Proceedings ICT 98. XVII International Conference on, 1998.

B. Poudel, Q. Hao, Y. Ma, Y. Lan, A. Minnich, B. Yu, X. Yan, D. Wang, A. Muto, D. Vashaee, X. Chen, J. Liu, M. S. Dresselhaus, G. Chen, Z. Ren, Science 2008, 320, 634.

S. Walia, S. Balendhran, H. Nili, S. Zhuiykov, G. Rosengarten, Q. H. Wang, M. Bhaskaran, S. Sriram, M. S. Strano, K. Kalantar-zadeh, Prog. Mater Sci. 2013, 58, 1443. 
[7] B. T. McGrail, A. Sehirlioglu, E. Pentzer, Angew. Chem. Int. Ed. 2015, 54, 1710.

[8] O. Bubnova, Z. U. Khan, A. Malti, S. Braun, M. Fahlman, M. Berggren, X. Crispin, Nat Mater 2011, 10, 429.

[9] G. H. Kim, L. Shao, K. Zhang, K. P. Pipe, Nat Mater 2013, 12, 719.

[10] V. Andrei, K. Bethke, F. Madzharova, S. Beeg, A. Knop-Gericke, J. Kneipp, K. Rademann, Adv. Eletron. Mater 2017, DOI: 10.1002/aelm.201600473.

[11] R. Hu, B. A. Cola, N. Haram, J. N. Barisci, S. Lee, S. Stoughton, G. Wallace, C. Too, M. Thomas, A. Gestos, M. E. d. Cruz, J. P. Ferraris, A. A. Zakhidov, R. H. Baughman, Nano Letters 2010, 10, 838.

[12] E. D. Eastman, Journal of the American Chemical Society 1928, 50, 283.

[13] R. E. L. Howard, A. B., Discussions of the Faraday's society 1957, 23, 113.

[14] S. M. Girvin, J Solid State Chem 1978, 25, 65.

[15] H. J. V. Tyrrell, Taylor, D. A., Williams, C. M., Nature 1956, 177, 2.

[16] B. R. Brown, M. E. Hughes, C. Russo, Phys Rev E 2004, 70.

[17]

[18]

[19]

A. E. Aliev, I. N. Kholmanov, P. K. Khabibullaev, Solid State Ionics 1999, 118, 111.

Y. Marcus, Chem Rev 2009, 109, 25.

J. Agar, C. Mou, J. L. Lin, The Journal of Physical Chemistry 1989, 93, 2079.

M. Bonetti, S. Nakamae, M. Roger, P. Guenoun, J. Chem. Phys. 2011, 134.

M. Bonetti, S. Nakamae, B. T. Huang, T. J. Salez, C. Wiertel-Gasquet, M. Roger, J. Chem. Phys. 2015, 142, 244708.

N. S. Hudak, G. G. Amatucci, J Electrochem Soc 2011, 158, A572.

Y. Akgöl, C. Cramer, C. Hofmann, Y. Karatas, H.-D. Wiemhöfer, M. Schönhoff, Macromolecules 2010, 43, 7282.

[24] J. R. Varcoe, Physical Chemistry Chemical Physics 2007, 9, 8.

[25] O. Larsson, E. Said, M. Berggren, X. Crispin, Adv. Funct. Mater. 2009, 19, 3334.

H. Wang, U. Ail, R. Gabrielsson, M. Berggren, X. Crispin, Adv. Eng. Mater. 2015, 5, 6.

[27] D.-W. Oh, Jain, A., Eaton, J. K., Goodson, K. E., Lee, J. S. , International Journal of Heat and Fluid flow 2008, 29, 1456.

[28] F. Toribio, J. Bellat, P. Nguyen, M. Dupont, J. Colloid Interface Sci. 2004, 280, 315.

[29] C. A. N. Decastro, S. F. Y. Li, A. Nagashima, R. D. Trengove, W. A. Wakeham, J Phys Chem Ref Data 1986, 15, 1073.

[30] O. Bubnova, in Linköping Studies in Science and Technology, Vol. PhD, Linköping University, Sweden, Linköping University Press 2013, 53.

[31] O. Larsson, X. Wang, M. Berggren, X. Crispin, Sensors and Actuators B: Chemical 2010, $143,482$. 
a.

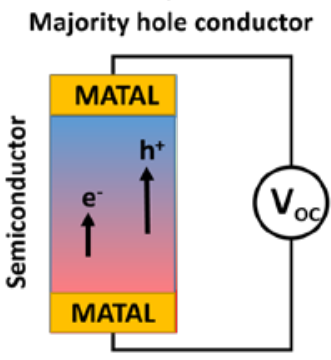

d. b. Holes pass in external circuit

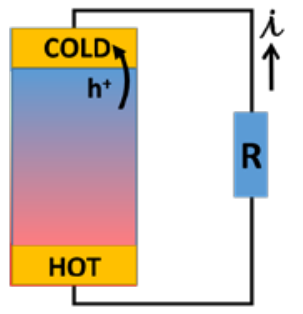

e.
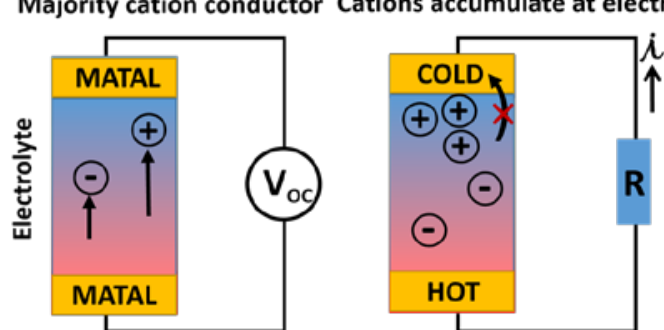

c.

Current remains constant

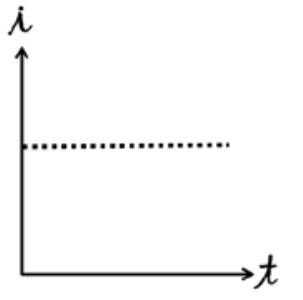

f.

Current decays with time

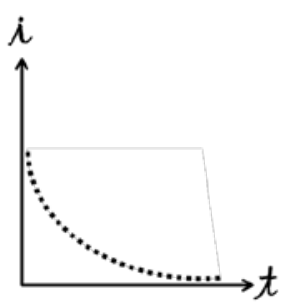

Figure 1 | Thermoelectric effect with electronic charge carriers in a semiconductor and ionic charge carriers in an electrolyte

a. and b. schematically demonstrate the electronic conductors displaying majority hole $\left(\mathrm{h}^{+}\right)$conduction. c.The thermoelectric generator (TEG) with electronic Seebeck effect can provide a continuous output current and power. d. The ionic conductors with majority cations (+) conduction. e. and f. show that ions accumulating at the metal electrodes induce a transient thermo-induced current that decreases to zero over time. 


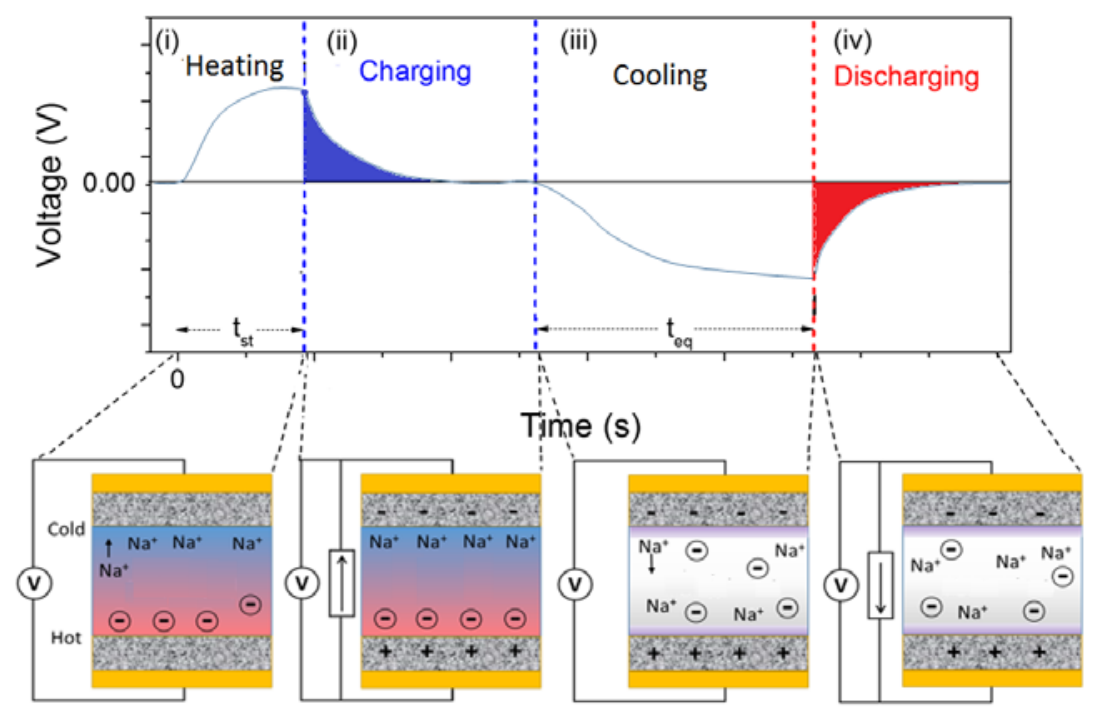

Figure 2 | Operation principle of ionic thermoelectric supercapacitor (ITESC).

Voltage curve and mechanism sketch of a full charge and discharge cycle: (i) establishing the temperature gradient through heating leads to an ionic thermovoltage, (ii) thermoelectric charging of the supercapacitor, (iii) cooling to reach equilibration with $\Delta T=0$, and (iv) discharging. 

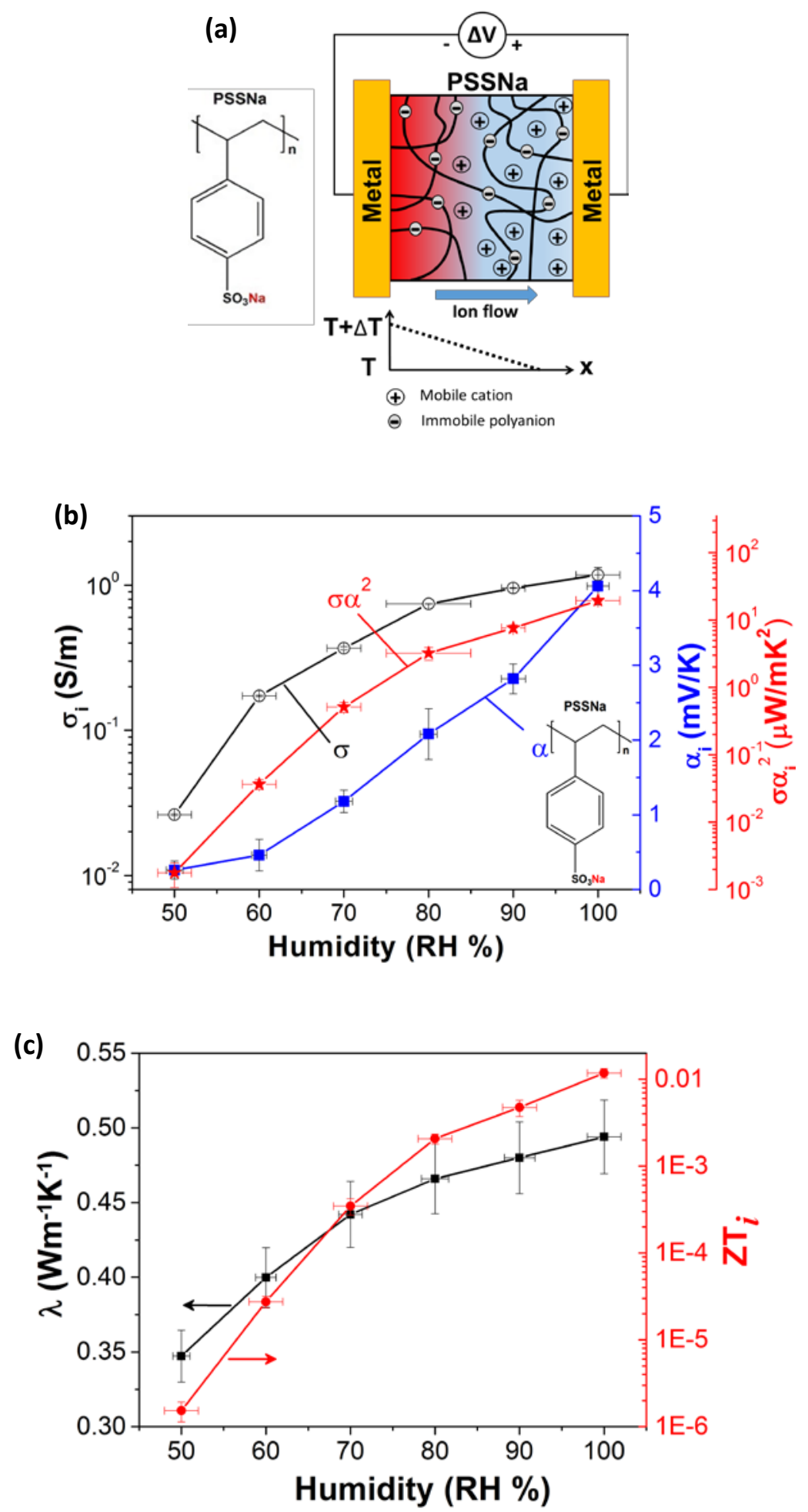

Figure 3 | Thermoelectric properties of polyelectrolytes versus humidity.

(a) Chemical structure of PSS:Na and sketch of the thermodiffusion of cations in a polyanion submitted to a temperature difference. For PSS:Na, the mobile ions $\left(\mathrm{Na}^{+}\right)$diffuse to the cold side. (b) Evolution of the ionic conductivity $\left(\sigma_{\mathrm{i}}\right)$, Seebeck coefficient $\left(\alpha_{\mathrm{i}}\right)$ and corresponding power factor $\left(\sigma \alpha_{\mathrm{i}}{ }^{2}\right)$ for PSS:Na versus RH. (c) The evolution of the thermal conductivity $(\lambda)$, and $\mathrm{ZT}_{\mathrm{i}}$ versus RH. All measurements were done at room temperature 
(a)
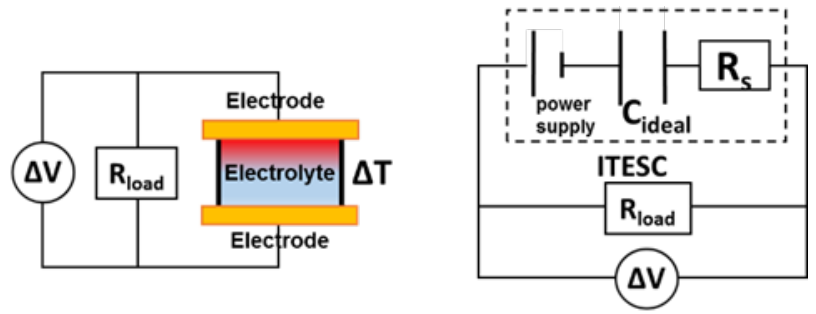

(b)

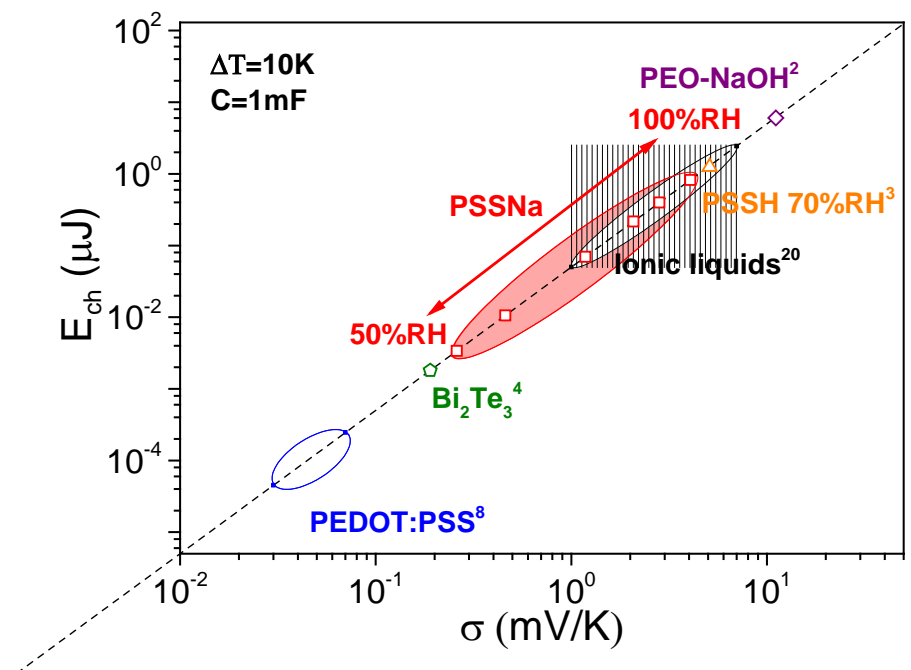

(c)

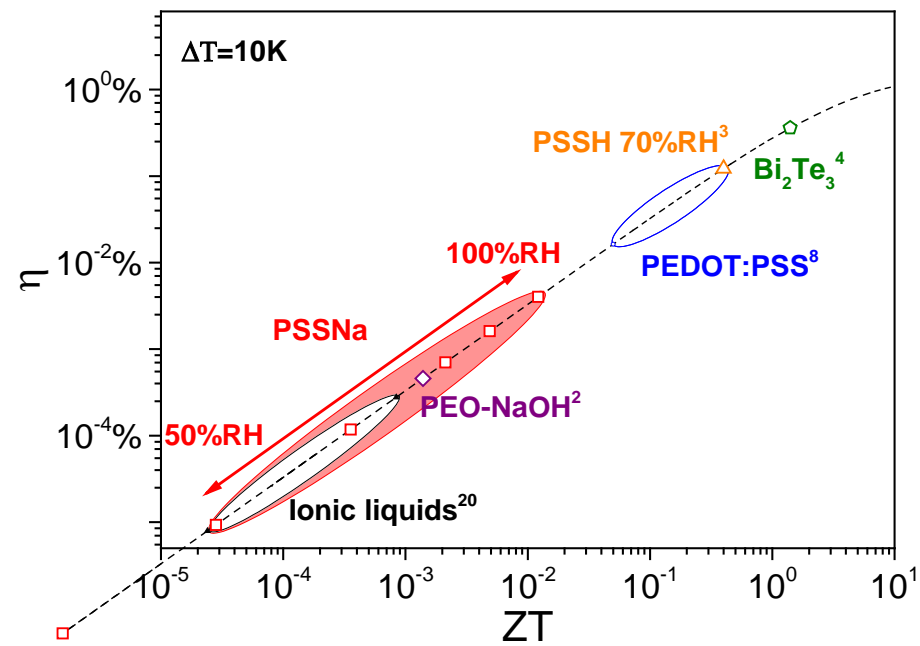

Figure $4 \mid$ Energy of charging and efficiency for PSS:Na and other thermoelectric materials.

(a) (left) Measurement set-up and (right) the equivalent circuit of ITESC in the experimental set-up. (b) The energy transfer to supercapacitor from charging of different thermoelectric material $(\Delta T=10 \mathrm{~K}, \mathrm{C}=1 \mathrm{mF})$. For ionic matierls (PSS:Na, PSSH, PEO-NaOH and ionic liquids) the structure of device is ITESC, and for electronic 
material (PEDOT and $\mathrm{Bi}_{2} \mathrm{Te}_{3}$ ) the structure of device is by connecting the TEG with a supercapacitor as shown in figure (a). (c) The efficiencies of materials with different ZT. 